\title{
A LOW POWER HIGH ACCURACY CMOS TIME-TO-DIGITAL CONVERTER
}

\author{
Poki Chen, Shen-Iuan Liu, and Jingshown Wu \\ Department of Electrical Engineering, National Taiwan University, Taipei, Taiwan China
}

\begin{abstract}
In this paper, we present a new CMOS time-todigital converter (TDC) with the cyclic delay line structure. The static supply current is $2-n A$ only. Furthermore, the continuous calibration is no longer needed. The TDC can be shunt down between measurements to make the power consumption negligible. The circuit with 64 -stage cyclic delay line has been fitted into $0.25 \mathrm{~mm} \times 0.75 \mathrm{~mm}$ chip area with a typical $0.8-\mu \mathrm{m}$ SPDM process. The measured resolution is 286 picoseconds, and the measured single-shot accuracy is less than 143 picoseconds. Both can be made much less if the control voltage is well tuned.
\end{abstract}

\section{INTRODUCTION}

Time interval digitization is an important building block for many instrumentation circuits, such as range finder, phase meter, robot vision, PM demodulator, etc.[1-3] Due to its large power consumption and circuit size, the conventional TDC with subnanosecond resolution can hardly be implemented in the portable systems[4]. At the end of 1995, a novel TDC circuit, named linear TDC here due to its linear delay line structure, is proposed to get 780 picoseconds resolution (after averaging hundreds of measurements) with $15 \mathrm{~mW}$ power consumption[5]. The portability problem is thus solved at the expense of $3 n s$ single shot accuracy. Figs. 1a and $1 \mathrm{~b}$ show the block diagrams of the circuit and the pulseshrinking delay element.

Basically, the pulse-shrinking delay element is a signal buffer composed by two NOT gates. The maximum conductible current of the first NOT gate is controlled by the bias voltage $\mathrm{V}_{\text {bias }}$. The degree of width shrinking of the input pulse can be easily controlled through adjusting $\mathrm{V}_{\text {bias }}$. A reference pulse with duration $T_{\text {ref }}$ is fed into the delay line for calibration. The delay-locked loop, cooperating with another delay line of same size, will adjust $\mathrm{V}_{\text {bias }}$ to make the reference period disappear ex- actly in the last delay element. In the measurement, the input period $T_{i n}$ with $T_{i n} \leq T_{r e f}$ will be fed into the delay line instead. Suppose that $T_{\text {in }}$ disappears in the $n$-th delay element, then the width is measured as $n \times T_{r e f} / N$, where $N$ is the number of elements in the delay line. For a $20 \mathrm{MHz}$ reference clock and a delay line of 64 elements, the resolution is $50 n s / 64=780 p s$ theoretically.

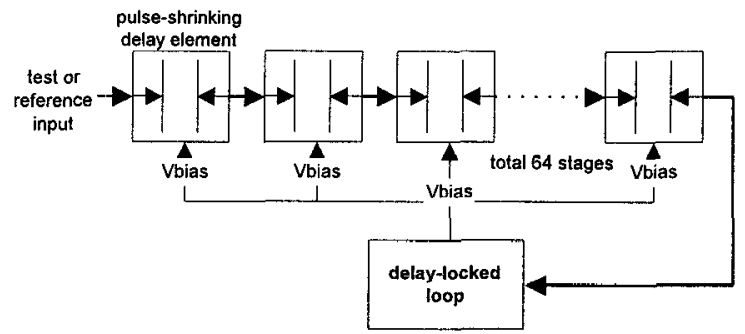

(a)

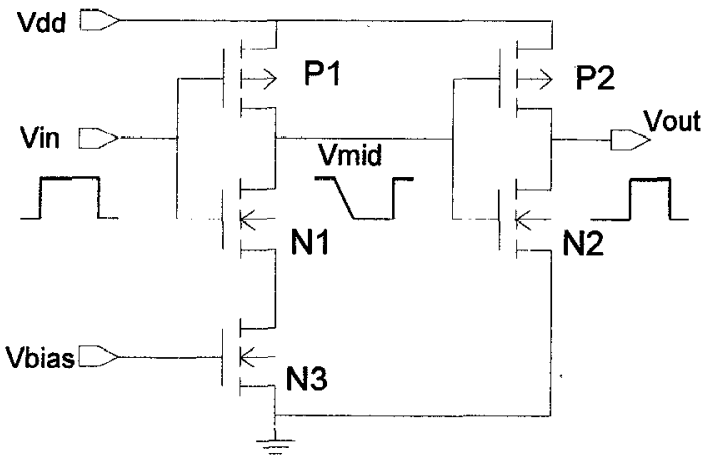

(b)

Fig. 1a The block diagram of the linear TDC

1b The pulse-shrinking delay element

This circuit established a milestone of low-cost, high-accuracy portable TDC systems. But there are still some important features needed to be improved. Due to many unavoidable variations $[5,6]$, the calibration of the TDC must be done continuously to keep $T_{r e f}$ just disappear in the last element of the delay line. This consumes much system power, and the circuit can not be shunt down between measurements for power savings. Also, 
the mismatch among pulse-shrinking delay elements results in poor delay line linearity. This sets a serious limit to the TDC resolution. When higher TDC resolutions are required, the pulse-shrinking delay line must be lengthened to accommodate more delay stages. And the linear TDC will become too long and must be folded into 2 or more segments due to reasonable chip width or length limit. The inter-segment wiring is much longer than the intra-segment one[6]. It causes large nonlinearity error and further limits the resolution of the TDC to be 6- to 7-bits only.

Finally, the input pulse shrink more rapidly at the last few elements, because it will become too short to drive the next stage to toggle its state. For the TDCs with coarse resolution, this phenomenon may not be noticeable: But for the TDCs with deep sub-nanosecond resolution, this kind of nonlinearity will dominate and deteriorate the accuracy. To overcome all these problems, a new TDC circuit is proposed in this paper. The circuit has been implemented by a $0.8-\mu \mathrm{m}$ SPDM CMOS process.

\section{OPERATION PRINCIPLES}

The block diagram of the new TDC is shown in Fig. 2a. The input pulse circulates in the cyclic structure. It shrinks a certain amount in each cycle until it thoroughly diminished. Assume that the counter counts $N$ for the reference input $T_{r e f}$ and $n$ for the test interval $T_{i n}$, then $T_{i n}$ is measured as:

$$
T_{i n}=\frac{n}{N} T_{r e f}
$$

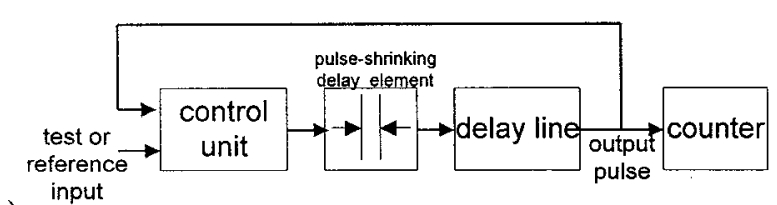

(a)

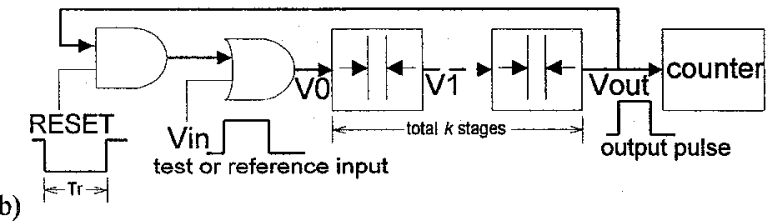

Fig. 2a The conceptual block diagram of the new TDC 2b The circuit of the new TDC in Fig. 2a

Practically, the pulse-shrinking delay elements can also served as delay units. So, the ideal delay line in Fig. 2a is replaced by series-connected pulse-shrinking delay elements as shown in Fig. 2b. Since the whole circuit of the new TDC is equivalently composed by even number of NOT gates, there are two stable states, $\mathrm{V}_{0}=\mathrm{V}_{1} \cdots=\mathrm{V}_{\text {out }}=$ LOW and $\mathrm{V}_{0}=\mathrm{V}_{1} \cdots=\mathrm{V}_{\text {out }}=\mathrm{HIGH}$. Where $V_{i}$ is the output of the $i$-th element in the delay line. To make sure that the circuit settles in the proper stable state $V_{0}=V_{1} \cdots=V_{\text {out }}=\mathrm{LOW}$ from power on, an external control pin RESET must be added as shown in Fig. 2b. The degree of shrinking is controlled by the bias voltage $V_{\text {bias }}$ which can be adjusted to get over $10^{2}$ or much more cycles for the reference period $T_{r e f}$. The corresponding resolution can be extremely fine.

Because the element mismatch and intra-segment wiring mismatch discussed will be went through per cycle by the input pulse, there will not be any measurement nonlinearity from cycle to cycle. The linearity problem is solved successfully. In this new TDC, it is not necessary to keep the reference pulse $T_{\text {ref }}$ just disappeared at some specific cycle or stage. Only if the bias voltage $\mathrm{V}_{\text {bias }}$ is properly adjusted such that $T_{\text {ref }}$ circulates the delay line enough times before diminished out to keep sufficient resolution. The continuous calibration is no longer needed, and the circuit can be shunt down between measurements to save power. Also, the associate delay-locked loop and its cooperating delay line can be saved. This greatly simplify the TDC circuit.

Basically, the output bits of the new TDC are unlimited. It can be increased by merely adding the number bits of the internal counter in Fig. 2b. And the control of $\mathrm{V}_{\text {bias }}$ must be made accurate enough. On the contrast, the linear TDC [10] must double the size of its delay lines to increase merely one output bit. No averaging is needed to improve the resolution. Because the single-shot resolution itself is accurate enough. The nonlinearity, caused by the pulse shrinking out more rapidly at the last few stages, can be treated as a constant count offset for a given bias voltage $\mathrm{V}_{\text {bias. }}$. And it can be completely calibrated out by the following technique.

Offset Elimination: First, feed $T_{r e f}$ and $T_{r e} / 2$ into the TDC input. Assume that the circuit gets $N$ and $N^{\prime}$ counts respectively. Then

$$
\begin{aligned}
& T_{r e f}=\alpha N+T_{o f f s e t} \\
& \frac{T_{r e f}}{2}=\alpha N^{\prime}+T_{o f f s e t}
\end{aligned}
$$$$
\text { and }
$$

Where $\alpha$ is the effective resolution, and $T_{\text {offset }}$ is the measurement offset caused by faster shrinking just before the input pulse disappeared. 


$$
\begin{aligned}
\alpha & =\frac{T_{r e f}}{2\left(N-N^{\prime}\right)} \\
T_{\text {offser }} & =\frac{T_{r e f}\left(N-2 N^{\prime}\right)}{2\left(N-N^{\prime}\right)}
\end{aligned}
$$

If the calculated resolution $\alpha$ is above requirement, we must raise $\mathrm{V}_{\text {bias }}$ carefully to make $N$ large enough. This in turn lowers the effective resolution $\alpha$ to a satisfactory value as expected from eq. (4). Next, feed measured interval $T_{i m}$ into the TDC input, and assume that the counter result is $n$. Then, the measured width of $T_{\text {in }}$ becomes:

$$
T_{i n}=\alpha \times n+T_{\text {offset }}=\frac{T_{r e f}\left(n+N-2 N^{\prime}\right)}{2\left(N-N^{\prime}\right)}
$$

\section{EXPERIMENTAL RESULTS}

To demonstrate the feasibility of the proposed TDC, we have fabricated the circuit with a $0.8-\mu \mathrm{m}$ SPDM CMOS process. The new TDC is composed by 64 delay elements with $(\mathrm{W} / \mathrm{L})=3 \mu \mathrm{m} / 0.8 \mu \mathrm{m}$ for $\mathrm{P} 1$ and $\mathrm{N} 1$ transistors, $(\mathrm{W} / \mathrm{L})=7.6 \mu \mathrm{m} / 0.8 \mu \mathrm{m}$ for $\mathrm{P} 2$ transistor, $(\mathrm{W} / \mathrm{L})=2.3$ $\mu \mathrm{m} / 0.8 \mu \mathrm{m}$ for $\mathrm{N} 2$ transistor, and $(\mathrm{W} / \mathrm{L})=1.3 \mu \mathrm{m} / 0.8 \mu \mathrm{m}$ for N3 transistor in each pulse shrinking delay element. The photomicrograph of the new TDC is shown in Fig. 3. The size of circuit is reduced to $0.14 \mathrm{~mm} \times 0.75 \mathrm{~mm}$ (without the internal counter). If the internal counter is included also, the chip size is estimated to be $0.25 \mathrm{~mm} \times$ $0.75 \mathrm{~mm}$. All the experimental results presented here are collected from these prototype chips. The static dissipation current is less than $2 n A$, and the average current consumption is calculated to be less than $100 \mathrm{nA}$ from a 5- $V$ supply.

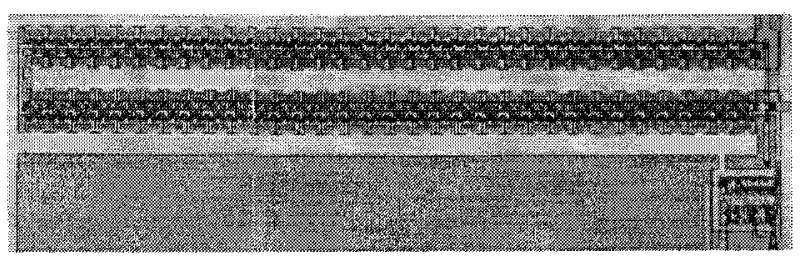

Fig. 3 The photomicrograph of the new TDC

Fig. 4a shows a typical output code distribution for 50 single-shot measurements of the new TDC. Compared with Fig. 4b, the typical distribution of single-shot measurements of the linear TDC[5], the single-shot measurement of the new TDC is very stable. No averaging is needed to improve the resolution. To explore the effective resolution and the measurement offset of the new TDC, a series of experiments with the same condition were conducted. The only parameter varied was the width of the input pulses generated by HP $8110 \mathrm{~A}$ pulse generator. The same input pulse widths were also measured with a Standford Research Systems SR620 universal counter. The measured results of single-shots along with the theoretical prediction line are depicted in Fig. 5a. It shows that the experimental data agrees with the prediction. From eq. (2), the effective resolution $\alpha$ is calculated to be $286 \mathrm{ps}$. Also, the measurement offset $T_{\text {offser }}$ is calculated to be $344 \mathrm{ps}$. This again confirms our inference that the input pulses shrink faster just before diminished. The dead time of single-shot measurements is at most a few microseconds. The measurement rate of at least $100 \mathrm{kHz}$ is promised. Fig. $5 \mathrm{~b}$ shows the error between single-shot measurements and the theoretical calculation. All the errors are below $1 / 2$ resolution (143 ps). This assures every output bit is valid.

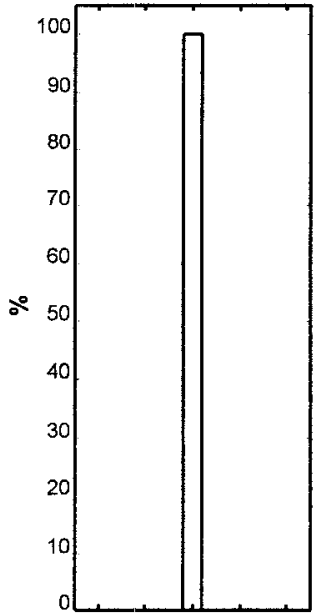

(a) output for the new TDC

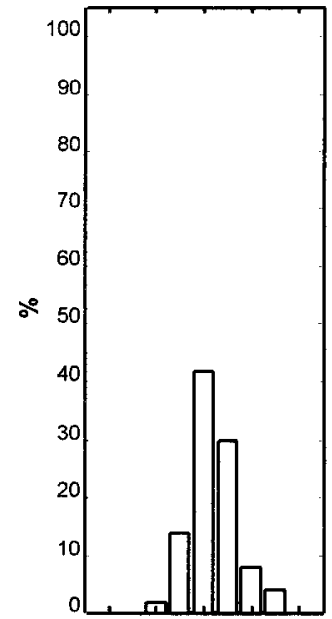

(b) output for the linear TDC
Fig. 4 Typical distribution of single-shot measurement results in 50 measurements for (a) the new TDC, (b) the linear TDC

The effective resolution and the single-shot accuracy can be further improved, if we control the bias voltage $\mathrm{V}_{\text {bias }}$ more accurately. By simulations, the resolution of the new TDC can be as low as tens of picoseconds or less. Table I shows a portion of simulation result for $\mathrm{V}_{\text {bias }}=3.10 \mathrm{~V}$ and $T_{i n}=6.0 \mathrm{~ns}$. The effective resolution is about $37.5 \mathrm{ps}$. 


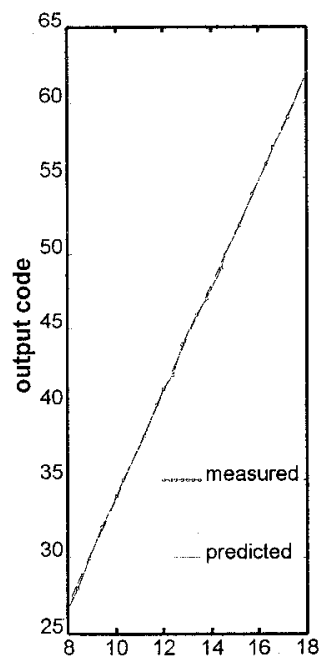

(a) input interval in $n s$

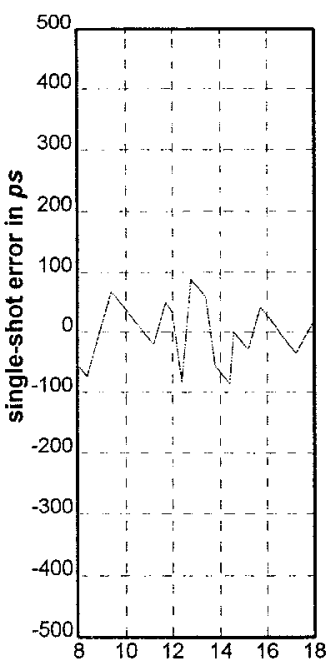

(b) input interval in ns
Fig. 5a The result of the single-shot measurements and the theoretical prediction line

$5 b$ The measured single-shot error

Table. I The beginning portion of the simulation result with $\mathrm{V}_{\text {bias }}=3.10 \mathrm{~V}, T_{\text {in }}=6 \mathrm{~ns}$.

\begin{tabular}{|l|c|c|c|c|c|}
\hline pulse no. & 1 & 2 & 3 & 4 & 5 \\
\hline width(ns) & 5.9758 & 5.9384 & 5.9008 & 5.8633 & 5.8258 \\
\hline shrinkage(ps) & & 37.4 & 37.6 & 37.5 & 37.5 \\
\hline & 6 & 7 & 8 & 9 & 10 \\
\cline { 2 - 6 } & 5.7884 & 5.7509 & 5.7135 & 5.6759 & 5.6385 \\
\cline { 2 - 6 } & $\mathbf{3 7 . 4}$ & $\mathbf{3 7 . 5}$ & 37.4 & $\mathbf{3 7 . 6}$ & $\mathbf{3 7 . 4}$ \\
\hline
\end{tabular}

\section{SUMMERY}

An extremely low-power and high-accuracy CMOS time-to-digital converter with the cyclic delay line structure has been presented. With the $0.8-\mu \mathrm{m}$ SPDM process and 64 delay elements, this new TDC can measure input interval up to $20 \mathrm{~ns}$ and reach a resolution of $286 \mathrm{ps}$ experimentally. The measured single-shot accuracy is less than $1 / 2$ resolution. No averaging is needed to improve accuracy. The TDC induces almost no extra cost for increasing the number of its output bits. When cooperated with TDCs using counter method, this TDCs can be used as interpolation circuits[7] to greatly increase the linear measurement range while keeping the same resolution. No continuous calibration is needed. This enables the TDC to be shunt down between measurements to save more battery power. This certainly opens another possible applications of the new TDC in low power and portable systems.

\section{ACKNOWLEDGMENT}

The authors would like to express their deep appreciation to Miin-Hwa Jiang of Center for Measurement Standards, Industrial Technology Research Institute for his measurement advising and instruments providing. The authors would like also to thank the National Science Council for financial supporting and thank the Chip Implementation Center (CIC), National Science Council, Taiwan, for the fabrication of the test chip. This work was sponsored by NSC-85-2622-E-002-010.

\section{REFERENCES}

[1] Määttä, J. Kostamovaara, M. Koskinen, and R. Myllylä, "Time-to-digital converter for fast, accurate laser range finding," in Proc. SPIE Industrial Inspection, Sept. 1988, vol. 1010, pp. 60-67.

[2] J. Kostamovaara, K. Määttä, M. Koskinen, and R. Myllylä, "Pulsed laser radars with highmodulation-frequency in industrial applications," in Proc. SPIE Laser Radar VII: Advanced Technol. Applicant., Jan. 1992, vol. 1633, pp.114-127

[3] Rapeli et al. "Method and circuitry for demodulation of angle modulated signals by measuring cycle time, "US Patent 5,270,666, Dec. 1993.

[4] T. Otsuji, "A picosecond-accuracy, 700-Mhz range si-bipolar time interval counter LSI," IEEE J. Solid-State Circuits, vol. 28, pp.941-947, Sept. 1993.

[5] Elvi Räisänen-Ruotsalainen, etc. "A Low-Power CMOS Time-to-Digital Converter," IEEE JSSC, vol. 30, No. 9; pp. 984-990, Sept. 1995.

[6] Timo E. Rahkonen, etc. "The Use of Stabilized CMOS Delay Lines for the Digitization of Short Time Intervals," IEEE JSSC, vol. 28, No. 8, pp. 887-894, Aug. 1993.

[7] R. Nutt, "Digital time intervalometer," Rev. Sci. Instrum., vol. 39, no. 9, pp. 1342-1345, 1968. 